3

\section{Mast seeding promotes evolution of scatter-hoarding}

Running head: Masting and the evolution of seed caching

Rafał Zwolak ${ }^{1}$, Dale Clements ${ }^{2}$, Andrew Sih², and Sebastian J. Schreiber ${ }^{2}$

${ }^{1}$ Department of Systematic Zoology

Institute of Environmental Biology

Adam Mickiewicz University

Umultowska 89

61-614 Poznań, Poland

${ }^{2}$ Center of Population Biology

University of California

One Shields Avenue

Davis, California, 95616 USA

KEYWORDS (4-6): Evolutionary stable strategies, masting, scatterhoarding, pilferage, seed caching, synzoochory. 


\section{ABSTRACT}

Many plant species worldwide are dispersed by scatterhoarding granivores: animals that hide seeds in numerous, small caches for future consumption. Yet, the evolution of scatterhoarding is difficult to explain because undefended caches are at high risk of pilferage. Previous models have attempted to solve this problem by giving cache owners unrealistically large advantages in cache recovery, by kin selection (but individuals that cache and those that pilfer are usually unrelated), or by introducing reciprocal pilferage of "shared" seed resources. However, the role of environmental variability has been so far overlooked in this context. One important form of such variability is masting, which is displayed by many plant species dispersed by scatterhoarders. We use a mathematical model to investigate the influence of masting on the evolution of scatter-hoarding. The model accounts for periodically varying annual seed fall, caching and pilfering behavior, and the demography of scatterhoarders. Masting, through its effects on population density, reduces cache pilferage and lowers the reproductive cost of caching (i.e. the cost of caching for the future rather than using seeds for current reproduction). These reductions promote the evolution of scatter-hoarding behavior especially when interannual variation in seed fall and the period between masting events are high. 
Many species of woody plants, particularly those that produce nuts and nut-like dry fruits, rely for seed dispersal on scatterhoarders: animals such as corvids, rodents, and certain marsupials that hide seeds for future consumption in numerous, small, widely-dispersed caches (Forget and Vander Wall 2001; Peserdorfer et al. 2016; Vander Wall et al. 2017). However, explaining the evolutionary advantage of this behavior has been difficult. The caches are undefended and often suffer very high rates of pilferage by con- and heterospecifics (Schmidt and Ostfeld 2008; Jansen et al. 2012; Zwolak et al. 2016; Dittel et al. 2017), thus scatter-hoarding appears vulnerable to cheating by non-caching pilferers (Anderson and Krebs 1978; Smulders 1998; Vander Wall and Jenkins 2003). How can scatterhoarding be advantageous under such circumstances?

This problem can be solved by invoking kin selection (but cache owners and pilferers are usually unrelated) or by giving cache owners a high advantage in cache recovery over naïve individuals (Anderson and Krebs 1978). Empirical estimates of the owner advantage are widely varied, but in most studies cache owners are 2-4 times more likely to recover their caches when compared with naïve individuals (Jacobs and Liman 1991; Jacobs 1992; Briggs and Vander Wall 2003; Thayer and Vander Wall 2005; Vander Wall et al. 2006; 2008; Hirsch et al. 2013; Gu et al. 2017; see also Steele et al. 2011). Yet, some of these estimates may be too high. First, many studies estimated the per capita advantage of owner versus pilferer, but under natural conditions there typically are many potential pilferers per each cache owner. Thus, caches are more likely to be emptied by a pilferer than by the owner even when per capita advantage of the latter is substantial. Second, most empirical studies have been short-term, with cache recovery examined over several days at most. Long-term (e.g. overwinter) removal rates might be very similar for cache owners and pilferers because cache locations are forgotten over time (Balda and Kamil 1992; Bednekoff et al. 1997; Barkley and Jacobs 1998). Over the long-term, cache owners will increasingly rely on olfaction or random search, just like naïve individuals do. As a consequence, the owners advantage appears to be 
insufficient to prevent cache loss to pilferage (2-30\% lost per day, according to a review by Vander Wall and Jenkins 2003).

Another putative solution to this puzzle is the reciprocal pilferage hypothesis (Vander Wall and Jenkins 2003; see also Smulders 1998). In principle, caching can represent an adaptive, stable strategy when all caches are reciprocally pilfered by scatter-hoarding animals with overlapping home ranges. Under this scenario, caches represent a collective resource used by selfish individuals (Vander Wall and Jenkins 2003). The reciprocal pilferage hypothesis predicts that animals are unlikely to avoid pilferage, but can compensate for it by pilfering caches of other individuals. As a corollary, individuals should invest in their pilfering tactics rather than in theft-reducing strategies (Vander Wall and Jenkins 2003).

Yet, many species of scatter-hoarding animals put considerable effort in behaviors apparently aimed to reduce pilferage (Dally et al. 2006a). This phenomenon has been particularly well-studied in corvids (e.g. Dally et al. 2006b; Shaw and Clayton 2013), but scatter-hoarding rodents also use a diverse array of theft-reduction strategies, including some that are costly in time and energy (e.g. transporting seeds further to reduce the density of caches: Galvez et al. 2009; Hirsch et al. 2012), some that are potentially dangerous (e.g. caching in open places where predation risk is higher, but the risk of pilferage is lower: Muñoz and Bonal 2011; Steele et al. 2013, 2015) and some that appear quite sophisticated (e.g. deceiving observers by making "fake" caches: Steele et al. 2008). This level of investment in pilferage prevention rather than pilferage tolerance is unexpected under the reciprocal pilferage hypothesis, and indeed has been interpreted as evidence against it (Leaver et al. 2007; Pravosudov et al. 2010).

However, existing models of scatter-hoarding have largely overlooked the role of environmental variability in the evolution of this behavior. Such variability is pervasive in ecosystems dominated by plants that produce dry, scatterhoarder-dispersed fruits because such plants usually show pronounced masting (Herrera et al. 1998; Vander Wall 2001). Examples of scatterhoarderdispersed masting plants can be found in the tropics (Norden et al. 2007; Mendoza et al. 2018), 

hoarding.

deserts (Meyer and Pendleton 2015; Auger et al. 2016), and in temperate zones (Koenig and Knops 2000; Schauber et al. 2002; Shibata et al. 2002). While studies of masting have often emphasized the benefit of masting to plants in terms of reduced per capita seed predation, masting also has important effects on consumer population dynamics that can feedback to affect the evolution of both masting and caching. In particular, masting has long been known to cause cycles of feast and famine that induce striking fluctuations in consumer population size (Ostfeld and Keesing 2000; Yang et al. 2010; Bogdziewicz et al. 2016). Typically, masting triggers a temporary increase in consumer population size followed by a pronounced crash. Thus, when the next mast year comes, seed to consumer ratios are particularly high (Kelly 1994; Ostfeld and Keesing 2000).

We use a mathematical model to investigate the influence of mast-related fluctuations in scatterhoarder population size on the evolution of scatter-hoarding. The model accounts for periodic seed fall and demography of the scatter-hoarding species. The scatterhoarders consume or cache harvested seeds and pilfer or recover their own caches over years that differ in the magnitude of seed fall. Previous models demonstrated that caching is influenced by the owner's advantage in cache recovery and the probability that scatterhoarders survive long enough to use the caches (Anderson and Krebs 1978; Smulders 1998; Vander Wall and Jenkins 2003). Thus, we include these factors in our investigation, but expand on previous models by examining the effects of environmental variability resulting from mast seeding. We examine how the proportion of seeds scatter-hoarded versus immediately consumed varies with (1) masting intensity, (2) the frequency of mast years, (3) the owner's advantage in cache recovery, and (4) the survival of scatterhoarders. Our results demonstrate that mast-related fluctuations in scatterhoarder population size reduce the risk of cache loss to pilferers and the reproductive cost of caching (i.e. the cost of caching seeds for future 
We consider a population of scatterhoarders that experience three distinctive periods of seed

123

124 availability in each year. During the first period (fall), there is seed fall of $S(t)$ from the primary seed source. Seeds are gathered at a rate proportional to the density $n_{1}(t)$ of scatterhoarders during this period. The proportionality constant $a_{1}$ corresponds to the per-capita seed harvest rate. Seeds are also lost to other sources (e.g. competitors, germination, decay, etc.) at a per-capita rate of $L_{1}$. If all seeds are gathered or lost to other sources by the end of the first period, then the amount of seed gathered per individual equals:

All seeds above a threshold, T, are cached by individuals for later in the year. The seeds which are not cached, $\min \{G(t), T\}$, are used for survival and reproduction. The number of offspring produced by individual, $R_{1}(t)$, at the end of this first period is a decelerating function with a maximal number of offspring $b$ and a half saturation constant $h$ (i.e. $h$ is the amount of resources required to produce $b / 2$ offspring). The fraction of adults surviving from the first period (fall) to the second period of the year (winter/spring) equals $s_{1}$. Thus, the total density $n_{2}(t)$ of individuals entering the second period equals

$$
G(t)=\frac{a_{1} S(t)}{L_{1}+a_{1} n_{1}(t)}
$$$$
\text { quals }
$$

$$
n_{2}(t)=\left[R_{1}(t)+s_{1}\right] n_{1}(t) \text { where } R_{1}(t)=\frac{b \min \{G(t), T\}}{h+\min \{G(t), T\}}
$$

The main resource available to individuals during this second period is the total cache of seeds $\max \{G(t)-T, 0\} n_{1}(t)$. Owners of the cached seed who survived gather their cached seed at a rate proportional to the size of their cache. This proportionality constant $a_{2}$ corresponds to the per-capita rediscovery and use rate of their cache. All other individuals are assumed to pilfer seed from others' 
caches at a per-capita rate $a_{\text {pil. }}$ Seeds are lost to other sources at a per-capita rate of $L_{2}$. If all cached seeds are gathered or lost by the end of the second period, then the fraction of cache recovered by an individual that survived from period 1 to period 2 is

$$
M(t)=\frac{a_{2}}{L_{2}+a_{2}+a_{p i l}\left(n_{2}(t)-1\right)}
$$

while the fraction of this individual's cache that was pilfered by another individual is

$$
O(t)=\frac{a_{p i l}}{L_{2}+a_{2}+a_{p i l}\left(n_{2}(t)-1\right)}
$$

For seed cache whose owner died, the fraction that was recovered by a living non-owner is

$$
D(t)=\frac{a_{p i l}}{L_{2}+a_{p i l} n_{2}(t)}
$$

It follows that the amount of cached seed gathered by a surviving individual from period 1 is

$$
C_{\text {survivor }}(t)=\max \{G(t)-T, 0\}\left[M(t)+O(t)\left(s_{1} n_{1}(t)-1\right)+D(t)\left(1-s_{1}\right) n_{1}(t)\right]
$$

and the amount of cached seed gathered by a new individual from period 2 is

$$
C_{\text {new }}(t)=\max \{G(t)-T, 0\}\left[O(t) s_{1} n_{1}(t)+D(t)\left(1-s_{1}\right) n_{1}(t)\right]
$$

If $s_{2}$ is the fraction of individuals surviving to period 3 (summer), then the density of individuals entering period 3 equals

$$
n_{3}(t)=\left[R_{2}(t)+s_{2}\right] n_{2}(t)
$$

where $R_{2}(t)$ is the population-level per-capita fecundity corresponding to the weighted combination of reproductive contributions of individuals surviving from period 1 and new born individuals from period 1 (for simplicity, we assume the same value of $h$ for surviving and new born individuals):

$$
R_{2}(t)=\frac{b C_{\text {survivor }}(t)}{h+C_{\text {survivor }}(t)} \frac{s_{1}}{R_{1}(t)+s_{1}}+\frac{b C_{\text {new }}(t)}{h+C_{\text {new }}(t)} \frac{R_{1}(t)}{R_{1}(t)+s_{1}}
$$

During this final period of the year (summer), individuals rely on other resources with availability $A$ to reproduce and survive with probability $s_{3}$. Thus, the density of individuals entering the Fall of the next year equals

$$
n_{1}(t+1)=\left[R_{3}(t)+s_{3}\right] n_{3}(t) \text { where } R_{3}(t)=\frac{b A}{h+n_{3}(t)}
$$


By composing the equations across the three periods of the year, the yearly update rule for population densities at the beginning of Fall is

$$
n_{1}(t+1)=\left[R_{3}(t)+s_{3}\right]\left[R_{2}(t)+s_{2}\right]\left[R_{1}(t)+s_{1}\right] n_{1}(t)
$$

We modeled seedfall $\mathrm{S}(\mathrm{t})$ in the fall as a periodic function of time where the period $\mathrm{P}$ corresponds to the time between masting years. In the masting years, $S(t)=S_{\text {high }}$ and otherwise $S_{\text {low. }}$ Our analysis assumes that the total seed output, $\mathrm{S}(1)+\mathrm{S}(2)+\ldots+\mathrm{S}(\mathrm{P})$, is fixed and varies the proportion of total seed output in the masting year. Higher intensity of masting means more seeds during the masting year, but concomitantly fewer seeds in other years (as opposed to just increasing seed output in masting years with no effect on seed production in other years). Similarly, when we vary the number of years between masting events, the total seed output remains the same (i.e., longer intermast interval corresponds with higher seed production in mast years).

Figure 1 illustrates typical dynamics of the model for the baseline parameters described below. For all our simulations, the dynamics asymptotically exhibit stable cyclic behavior with a period corresponding to the period of the masting events. For low caching thresholds (dotted line in Fig. 1b), individuals cache most of their seeds every fall. For high caching thresholds (dashed line in Fig. 1b), individuals either only cache on masting years as shown or not at all (not shown). Population densities (Fig. 1c) exhibit seasonal as well as yearly variation. Moreover, for any given season, population densities are maximal following a masting event and decrease year to year until the next masting event. 


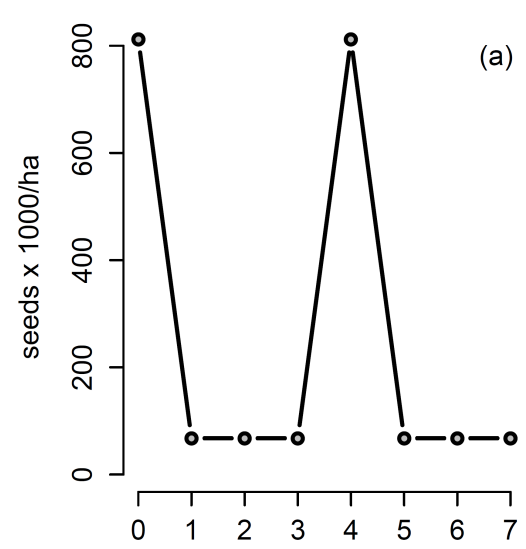

186

187

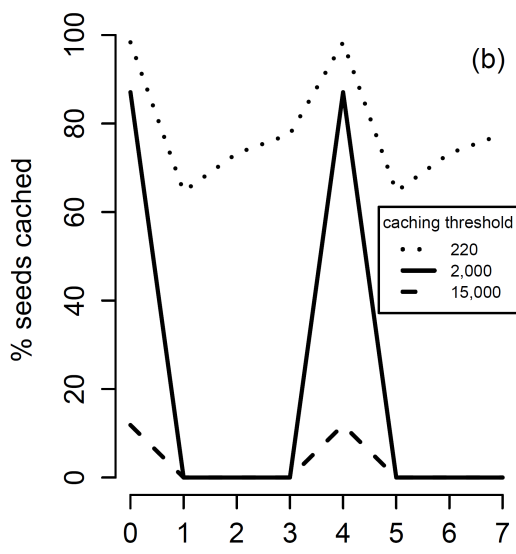

year

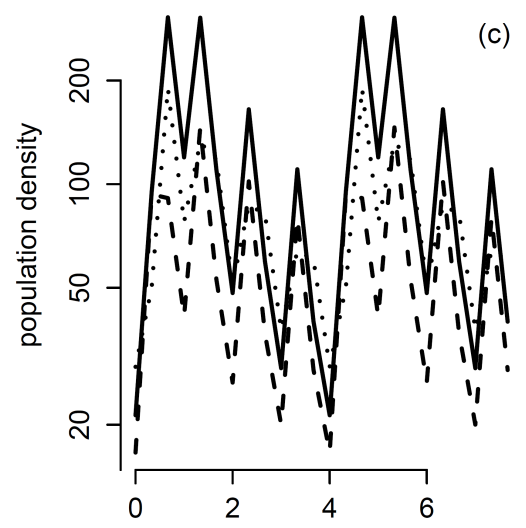

year

Fig. 1. The dynamics of fall seed masting (a), per-cent seed cached by an individual for different

caching thresholds $T(b)$, and population densities (c) for the different caching thresholds $T$ (see model description). In (a), there is a 4 year interval between fall masting events. In (b), the percent seeds cached by individuals for populations with a low (dotted), intermediate (solid), and high (dashed) caching threshold. In (c), the intra- and interannual variation in population densities for populations with a low (dotted), intermediate (solid), and high (dashed) caching threshold. The dynamics in (b) and (c) correspond to a stable 4-year periodic orbit of the model.

Model Parameters

The parameter values mimic an interaction between a masting tree and a scatterhoarding rodent and are based mostly on research on European beech (Fagus sylvatica) and yellow-necked mice (Apodemus flavicollis). Empirical results were used to generate order of magnitude parameter estimates, which were then adjusted, by no more than a factor of 10 , so that the equilibrium population sizes were roughly in line with those given in the literature. While we found it useful to base our parameter estimates on a specific, reasonably well-studied system, moderate variation in these parameters does not change the qualitative results.

The parameters $a_{1}$ and $L_{1}$ may be reduced to the single parameter $L_{1} / a_{1}$. Rearranging the equation for $G$ yields $L_{1} / a_{1}=\frac{\left(1-G / S_{0}\right) n_{1}}{G / S_{0}}$. The parameters $n_{1}$ and $n_{2}$ were taken to be the average 
species-wide density for Apodemus flavicollis: 17.7 individuals/ha (Jones et al. 2009 [PanTHERIA]). Estimates of the proportion of seeds removed from the forest floor $\left(G / S_{0}\right)$ tend to be variable with Zwolak et al. (2016) reporting 78\% seed removal during mast years and $91 \%$ seed removal during non-mast years for $A$. flavicollis, and Lelouarn and Schmitt (1972) reporting $41 \%$ and $74 \%$ seed removal by Apodemus sylvaticus during two different years. We selected the intermediate value of $74 \%$ as tentative estimate of seed removal. Thus, $L_{1} / a_{1}=6.22$. In all cases where $L_{1}$ and $a_{1}$ were treated as separate parameters, we used $a_{1}=1$ and set $L_{1}$ equal to our choice for $L_{1} / a_{1}$. The parameters $a_{\text {pil }}$ and $L_{2}$ may similarly be reduced to $L_{2} / a_{\text {pil }}$. Zwolak et al. (2016) estimated the recovery of seeds from artificial caches to be $54 \%$ during nonmast years and $5 \%$ during mast years, which we assumed to be roughly equivalent to the proportion of seeds recovered from an abandoned cache $(D / \max \{G(t)-T, 0\})$. We then estimated $L_{2} / a_{\text {pil }}$ to be between 15.1 and 336.3. In our analysis, we set this value to the upper end of this range (300) as this leads to more conservative estimates of when caching evolves. In all cases where $L_{2}$ and $a_{\text {pil }}$ were treated as separate parameters, we used $a_{p i l}=1$ and set $L_{2}$ equal to our choice for $L_{2} / a_{\text {pil }}$. We let $a_{2}=3$ (when $a_{p i l}=1$ ). This value approximates the results of several studies on scatterhoarding rodents (Vander Wall et al. 2006, 2008; Thayer and Vander Wall 2005; Hirsch et al. 2013) that documented seed removal rates by cache owners and naïve foragers. Although some research (Gu et al. 2017) suggests that the owner advantage might be even higher, it also might be lower, particularly later in the season, as we explained in the Introduction.

We assumed a maximum litter size of 11 individuals (Macdonald and Tattersall 2001), with one breeding event per period (2-3 litters per year: Pucek 1984). Assuming that half the population are female and half of the individuals born are female, this yields $b=5.5$.

We used Jensen's (1982) monthly winter survival estimate of $77.5 \%$ as the yearlong monthly survival rate (see also Pucek 1992 for similar values). We assumed that each period lasts four months, yielding $36.1 \%$ as the survival rate for each period $\left(s_{1}, s_{2}\right.$, and $\left.s_{3}\right)$. 

multiplied by half the maximum number of offspring $(b / 2)$. This value was calculated on the basis of energy contents of beech seeds (Grodziński and Sawicka-Kapusta 1970), energy requirements of yellow-necked mice $(0.60 \mathrm{kcal} / \mathrm{g} /$ day: Jensen 1982 ; average body mass of yellow-necked mice is 28.3 $\mathrm{g}$ : AnAge), and typical costs of reproduction-related energy expenditure in small mammals $(25 \%$ increase in energy expenditure during gestation and 200\% increase during lactation: Millar 1978; 1979; Gittleman and Thompson 1988; Sikes 1995; Zhu et al. 2015), given the length of gestation and lactation in yellow-necked mice (26 and 22 days, respectively: AnAge).

\section{Numerical Methods}

To identify the evolutionary stable caching strategies, we examined whether a small mutant subpopulation using the germination threshold $T_{m}$ can invade a resident population using the germination threshold $T$. When the mutant subpopulation densities $m_{i}(t)$ in each of the periods $i=1,2,3$ are sufficiently small, the density feedback of the mutant population on the resident population and itself is negligible. Hence, the dynamics of the mutant in the initial phase of invasion can be approximated by a linearization of its dynamics. We now describe these linearized dynamics. As the mutant and resident individuals only differ in their caching strategy, the amount of seeds gathered in year $t$ by a mutant individual equals the amount of seeds gathered $G(t)$ by a resident individual. As for the resident dynamics, yearly update of the mutant's fall density is of the form

$$
m_{1}(t+1)=\left[Q_{3}(t)+s_{3}\right]\left[Q_{2}(t)+s_{2}\right]\left[Q_{1}(t)+s_{1}\right] m_{1}(t)
$$
only differs from the resident in its threshold $T_{m}$

$$
Q_{1}(t)=\frac{b \min \left\{G(t), T_{m}\right\}}{h+\min \left\{G(t), T_{m}\right\}}
$$
the fall and individuals born in the fall: 


$$
Q_{2}(t)=\frac{b C_{m, \text { survivor }}(t)}{h+C_{m, \text { survivor }}(t)} \frac{s_{1}}{Q_{1}(t)+s_{1}}+\frac{b C_{\text {new }}(t)}{h+C_{\text {new }}(t)} \frac{Q_{1}(t)}{Q_{1}(t)+s_{1}}
$$

258

259 where $Q_{2}(t)$ differs from $R_{2}(t)$ only in its first term due to surviving individuals with the mutant caching strategy: $C_{m, \text { survivor }}(t)=\max \left\{G(t)-T_{m}, 0\right\} M(t)+\max \{G(t)-T, 0\}\left[O(t)\left(s_{1} n_{1}(t)-\right.\right.$ $\left.1)+D(t)\left(1-s_{1}\right) n_{1}(t)\right]$. Finally, the number of offspring produced by a mutant over the summer is the same as the resident i.e. $Q_{3}(t)=R_{3}(t)$.

Whether the mutants playing strategy $T_{m}$ are able to invade the residents playing the strategy $T$ or not depends on their long-term per-capita growth rate

$$
s\left(T, T_{m}\right)=\lim _{t \rightarrow \infty} \frac{1}{t} \log \left[Q_{3}(t)+s_{3}\right]\left[Q_{2}(t)+s_{2}\right]\left[Q_{1}(t)+s_{1}\right]
$$

provided the limit exists. Over the parameter space (see previous section) that we simulated, the population dynamics always converged to a periodic solution whose period $k P$ is a multiple $k$ of the seed masting period $P$. Typically, this multiple was 1 or 2 or 4 , the latter two corresponding to perioddoubling bifurcations. We developed R code to efficiently approximate these periodic solutions. For these periodic solutions of the resident dynamics, the long-term per-capita growth rate of mutant strategy $T_{m}$ against resident strategy $T$ equals

$$
s\left(T, T_{m}\right)=\frac{1}{k P} \sum_{t=1}^{k P} \log \left[Q_{3}(t)+s_{3}\right]\left[Q_{2}(t)+s_{2}\right]\left[Q_{1}(t)+s_{1}\right]
$$

A strategy $T$ is an ESS for caching if $s\left(T, T_{m}\right)<0$ for all strategies $T_{m} \neq \mathrm{T}$. Using the method of adaptive dynamics (Geritz et al. 1997), we identified potential ESSs by solving for roots of the fitness gradient $\frac{\partial s}{\partial T_{m}}(T, T)$. In the Appendix, we derive an explicit expression of this fitness gradient 


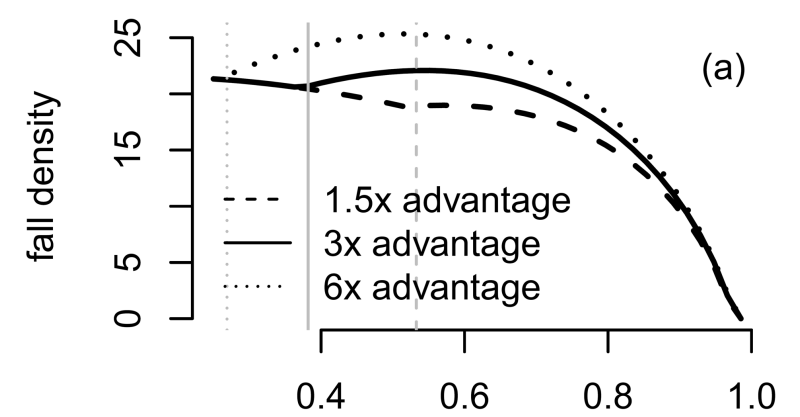

$\%$ seed during masting year

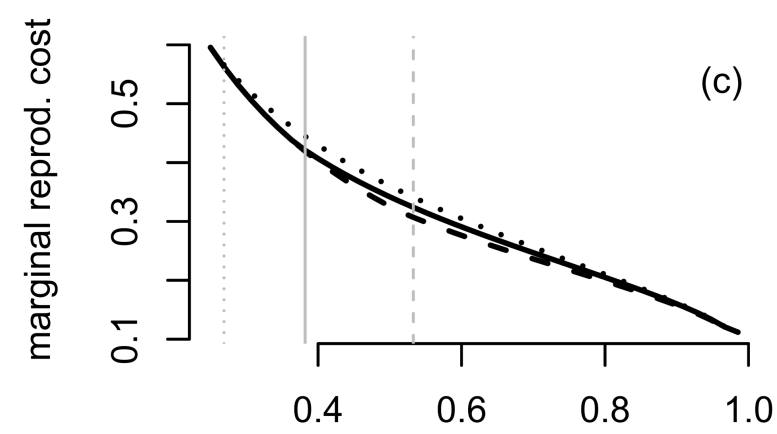

$\%$ seed during masting year

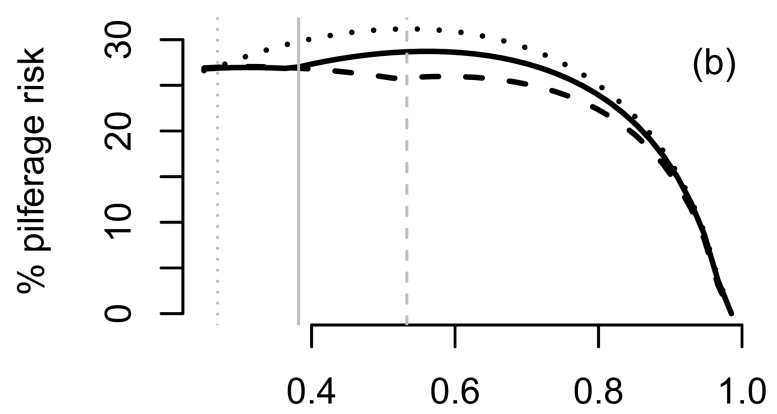

$\%$ seed during masting year

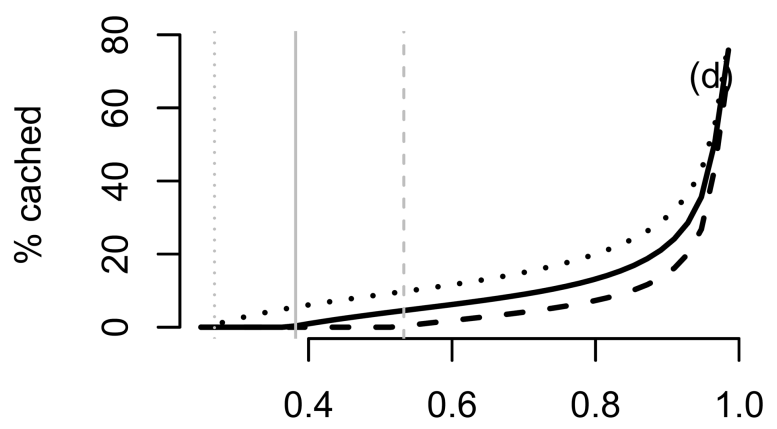

$\%$ seed during masting year

Fig. 2. Period 1 (fall) population density in individuals/ha (a), pilferage risk, defined as the probability

that cached seed would be pilfered (b), marginal reproductive costs of caching (c), and proportion of seeds cached rather than eaten (d) as a function of masting intensity (expressed as the percentage of total seed production that occurs during mast years), with mast occurring every $4^{\text {th }}$ year. Dashed, solid, and dotted lines represent the magnitude of owner's advantage in cache recovery (owners 1.5, 3, and 6 times more likely to discover their own caches relatively to naïve foragers). Plotted variables represent values in the mast year. Vertical gray lines correspond to the minimal masting intensity at which caching is selected for.

\section{Results}

Increasing intensity of masting generally results in decreased period 1 (fall) scatterhoarder population density (i.e., the density that enters masting events; Fig. 2a). Intuitively, because reproduction is a saturating function of seeds gathered, the reproductive gains of higher seed availability during masting years were outweighed by the reproductive losses due to lower seed 
availability during non-mast years. As seed caching generally increases fall population densities by preventing population crashes in winter/spring, this indirect positive effect of masting intensity appears when masting intensities is just sufficient to select for caching and results in intermediate positive trends in fall density (Fig. 2a).

Increasing masting intensity also reduces the risk that a cached seed would be pilfered (Fig.

$2 \mathrm{~b})$, particularly when $80 \%$ or more seeds are produced during mast years. Notice that the responses of pilferage risk and fall density are tightly correlated (see, also, Figs. 3 and 4). Furthermore, increasing masting intensity is associated with a decline in marginal reproductive costs of caching (Fig. 2c) and with an accelerating increase in the ESS proportion of seeds cached rather than eaten (Fig. 2d). In the baseline scenario, scatterhoarders start caching when about $50 \%$ of seeds are produced during mast years.

Higher recovery advantage tends to increase scatterhoarder densities (Fig. 2, dotted vs. solid vs. dashed line), probably because (i) on average individuals are recovering more of the cached seeds, and (ii) there is selection for increased seed caching (Fig 2d), which tends to be ecologically advantageous. Increasing the owner's advantage in cache recovery (Fig. 2, dotted line) selects for more caching despite increases in population densities and cache pilfering; decreasing the owner's advantage (Fig. 2, dashed line) decreases the proportion of seeds cached even though population densities and cache pilferage also drop. However, the effect of the owner's advantage in cache recovery is relatively weak compared to the effect of masting intensity. 


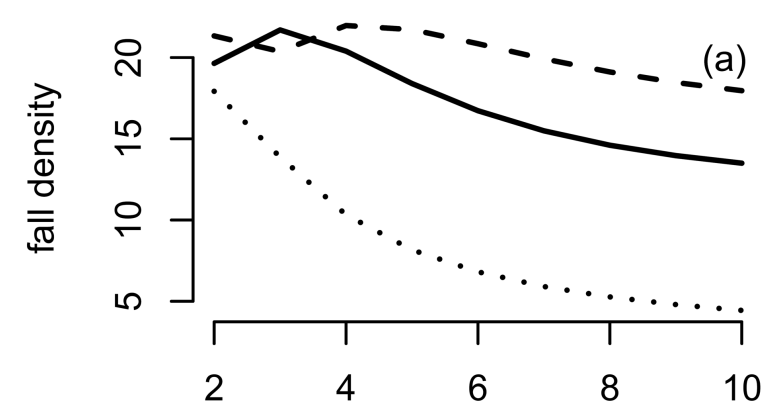

years between masting events

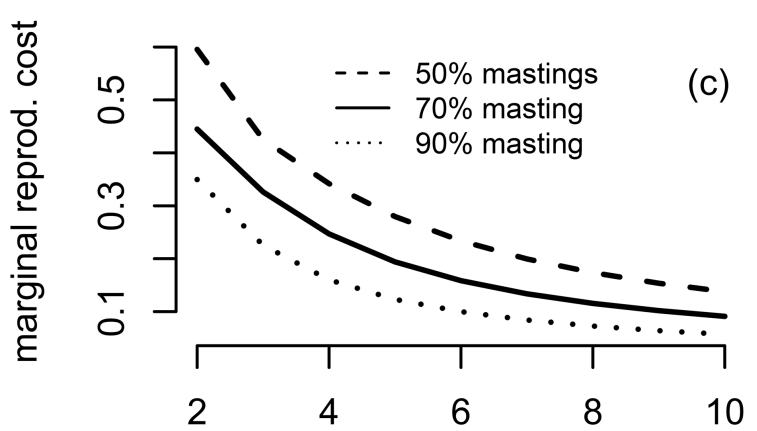

years between masting events

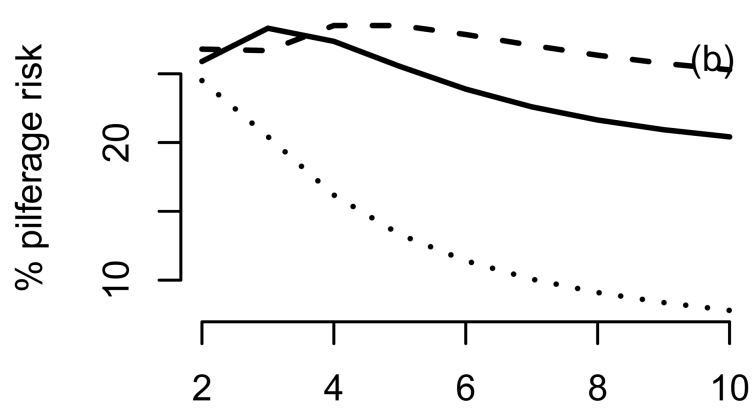

years between masting events

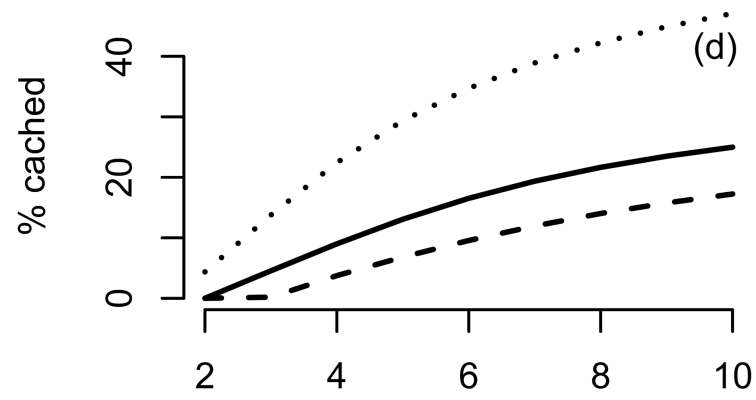

year between masting events

Fig. 3. Period 1 (fall) population density (a), pilferage risk (b), marginal reproductive costs of caching

(c), and proportion of seeds cached rather than eaten (d) as a function of masting interval. Dashed,

during mast years). Plotted variables represent values in the mast year.

Increasing the inter-mast period leads to declines in fall density (Fig. 3a), pilferage risk (Fig. 3b), and marginal reproductive costs (Fig. 3c), and an increase in the proportion of seeds cached (Fig. 3d). Intuitively, more years with poor seed crops between masting events leads to lower densities of individuals entering the fall of a masting year. This reduction in density leads to fewer individuals entering winter and, thereby, lower risk of seed pilferage. In addition, the low densities of individuals entering the masting years and the greater amount of seeds available during the masting year

321 increase the amount of seeds harvested per individual and, due to the effects of diminishing returns, lower marginal reproductive costs of caching. Collectively, lower reproductive costs and lower pilferage risk select for more caching. Varying masting intensity $(50,70$ or $90 \%$ of seeds produced 
during mast years: dotted, solid or dashed line on Fig. 3) affects the magnitude of these changes,
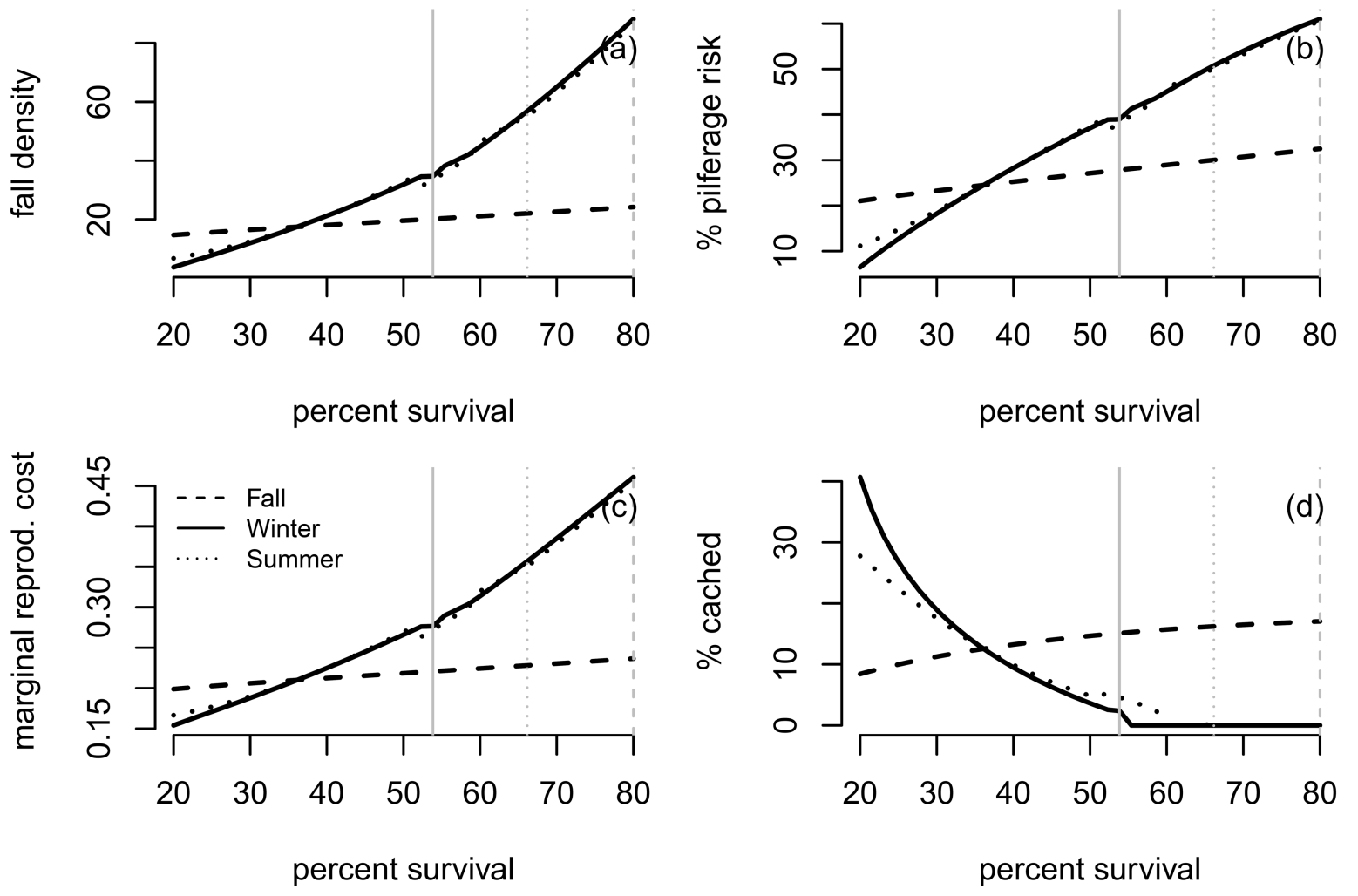

Fig. 4. Period 1 (fall) population density (a), pilferage risk (b), marginal reproductive costs of caching

(c), and proportion of seeds cached rather than eaten (d) as a function of scatterhoarder survival.

Dashed, solid, and dotted lines denote responses to changes in fall, winter, and summer survival,

maximal survivorships at which caching is selected for.

Increasing the survival of scatterhoarders leads to nearly identical increases in period 1 population

density, pilferage risk, and marginal reproductive costs of caching (Fig. 4). These effects are

considerably stronger for changes in winter and summer survival rather than in fall survival.

337 Intuitively, this is because the greatest concentration of births occurs between the fall and winter, 
339 to mortality during the previous period) than the summer and fall populations. Thus, an increase in

340 mortality in the fall affects a smaller proportion of the population than an increase in mortality in the

341 winter or summer. Interestingly, varying summer and winter/spring versus fall survival has

342 contrasting effects on the percentage of seeds cached. Increasing summer and winter/spring survival

343 leads to a sharp decline in the predicted proportion of seeds cached most likely due to the large

344 increase in fall density, and the drastically increased costs associated with increased pilferage risk

345 and lost reproductive opportunities. When monthly winter/spring and summer survival exceeded

$34650 \%$, no seeds should be cached. In contrast, increasing fall survival selects for an increase in the

347 percentage of seeds cached rather than eaten, most likely because the advantage of surviving to

348 make use of one's cache outweighs the weak increases in costs. 


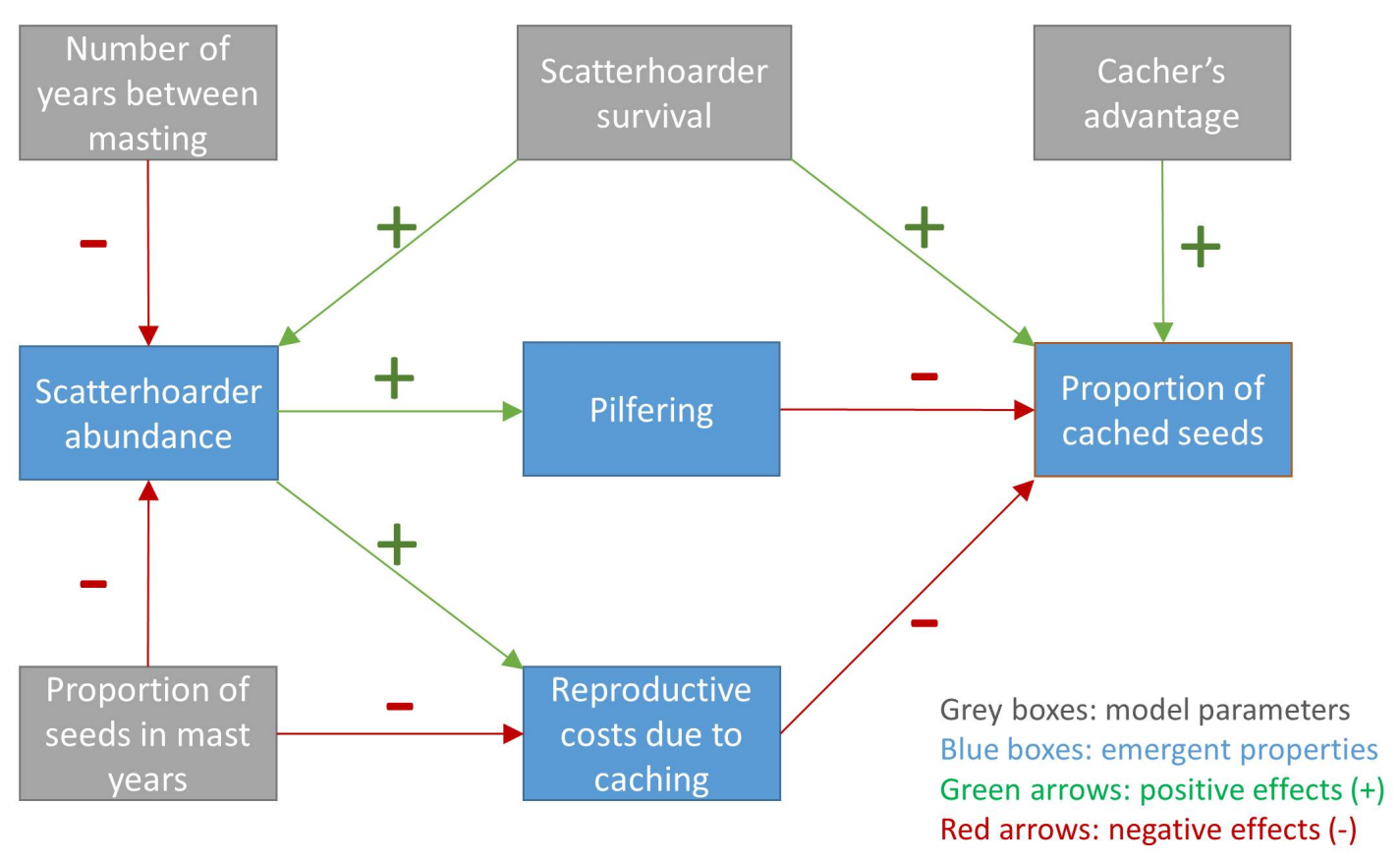

351 Fig. 5. Relationships between input parameters (masting interval and intensity, expressed as the proportion of seeds produced during mast years, scatterhoarder survival, and cacher's advantage in cache recovery) and emergent properties of the model (scatterhoarder population density, the proportion of cached seeds that are pilfered, reproductive costs of caching, and the quantity of interest: the proportion of seeds cached by scatterhoarders).

\section{Discussion}

The fact that masting causes strong fluctuations in populations of seed-eating animals has been wellknown for a long time (Curran and Leighton 2000; Ostfeld and Keesing 2000; Bogdziewicz et al. 2016), yet the traditional research focus has been on how the satiation-starvation cycle reduces seed losses to pre- and post-dispersal seed predators. More recently, researchers suggested that seed masting is one of the means by which plants manipulate behavior of their dispersers (Vander Wall 
2010). According to this reasoning, satiation of current energy needs induces granivores to cache seeds for future use (Vander Wall 2010). Here we show that the effects of masting on population dynamics and caching behavior are mutually dependent. By decreasing the degree of pilfering, the satiation-starvation cycle due to more extreme seed masting events may promote the evolution and maintenance of seed caching behavior. Thus, the decrease in seed predation, increase in per capita scatterhorder satiation, and reduction in pilfering pressure may each represent an important pathway by which the scatterhorder satiation-starvation cycle induced by masting may improve plant recruitment (Fig. 5). These nuanced interactions between plant and seed predator emphasize the importance of studying the feedbacks between population dynamics and behavioral evolution. Results of our simulations suggest that when seed production is highly variable, seed caching can evolve even when cache owners have little advantage over naive foragers in seed recovery (compare with Krebs and Anderson 1978). The costs of cache loss to pilferers are reduced in our model because periods of intense seed production coincide with low densities of scatterhoarders and thus few potential pilferers (see Dittel and Vander Wall 2018 for experimental data demonstrating that the magnitude of cache pilferage is determined by the abundance of scatterhoarders). When there is pronounced masting with relatively long intervals between masting events, densities of scatterhoarders entering the start of the next large masting event are low (Figs. 2 \& 3). Consequently, individuals are able to collect enough seeds to satiate their reproductive needs. As the yearly fitness is determined by the geometric mean of their fitness across the seasons and this geometric mean decreases with seasonal variation in fitness (Lewontin \& Cohen 1969; Gillespie 1977; Schreiber 2015), the benefits of reducing seasonal variation in fitness by increasing winter/spring reproduction outweigh the diminishing returns of increasing reproduction in the fall. Our results make a prediction that plants dispersed by scatterhoarders should have high interannual variation of seed production (typically measured with coefficient of variation, CV) relatively to plants dispersed by other means. This appears to be the case, at least when plants 
391

392

393

394

395

396

397

398

399

400

401

402

403

404

405

406

407

408

409

410

411

(endozoochorously) (Herrera et al. 1998). When explaining this pattern, researchers emphasized contrasting selective pressures acting on these groups of plants. Avoiding the risk of satiating frugivores was suggested as a factor that stabilizes seed production in plants dispersed endozoochorously. On the other hand, variable seed production in synzoochorous plants was interpreted as an adaptation that enabled reducing seed mortality caused by animals that act as seed predators and only incidentally disperse seeds (Herrera et al. 1998). However, we suggest that the high CV of plants dispersed by scatterhoarders can also be linked to the caching behavior of scatterhoarders.

There are several adaptive hypotheses of masting (e.g. increased pollination efficiency, predator satiation, and seed dispersal: reviews in Kelly 1994; Kelly and Sork 2002; Pearse et al. 2016) and these putative fitness benefits of masting can occur simultaneously. Thus, it is difficult to conclude whether variability in seed production evolved (at least partly) to stimulate more seed caching, or whether seed caching evolved in granivores that interacted with plants that already had high CV of seed production. Moreover, these two evolutionary pressures could co-occur, creating a positive feedback between the variability in plant production and granivore caching behavior. If, as our simulations suggest, masting intensity and mast interval are important for seed caching, then changes in plant masting patterns might affect the dynamics of seed caching, and therefore also the abundance of granivores and recruitment in plant populations. Our model is loosely based on the European beech - Apodemus mice system (Jensen 1982; Zwolak et al. 2016). Several studies have suggested that the European beech shows more frequent masting, probably due to global warming (Kantorowicz 2000; Schmidt 2006; Overgaard et al. 2007; Paar et al. 2011; but see Drobyshev et al. 2014). This could shift the beech-rodent interactions towards antagonism, with more seed consumed and fewer cached (Fig. 2). On the other hand, a recent meta-analysis of global data suggests that the interannual variation in seed crops is increasing (Pearse et al. 2017). Such a change could make seed caching more profitable for granivores. However, extreme interannual 
variation in seed crops might lead to a decline and even extinction in granivore populations, due to the difficulty in tracking resource levels (Fig 3).

Moreover, any environmental change that affects scatterhoarder population dynamics could alter caching behavior and, thereby, impact seed mortality. For example, we found that increased scatterhoarder survivorship during the winter or summer may select against caching behavior by increasing population densities entering the masting years (Fig. 4). Thus, changes in winter or summer conditions that are favorable for mice could harm seedling recruitment both directly by increasing seed predation and indirectly by discouraging seed caching. In contrast, the effects of improved conditions in the autumn on seedling recruitment is more difficult to predict as increased survivorship of scatterhoarders simultaneously selects for more caching and more seed predation due to higher population densities.

According to a recent review, at least 1,339 species of plants are dispersed by scatterhoarders (Gómez et al. 2018). Thus, good understanding of the evolution of caching behavior and of ecological determinants of seed caching is pivotal when our goal is to comprehend former selective pressures on synzoochorous plants, disentangle current mechanisms of seed dispersal, or to predict how global changes might alter seed dispersal patterns. Our study provides a step in this direction and suggests several promising avenues for future research. For example, future work should address the evolution of caching reaction norms instead of the simple threshold for caching considered here. Additionally, evolution of caching strategies could be different when individual variation in personalities or, more generally, phenotypes of seed dispersing animals (Zwolak 2018) is taken into account. Finally, comparing systems where reciprocal pilfering is common (e.g. rodents: Vander Wall and Jenkins 1998) versus those where it is rare (e.g. corvids: Pesendorfer et al. 2016) could provide further insights on the evolution of caching behavior. 


\section{Literature Cited}

Andersson, M., and J. Krebs. 1978. On the evolution of hoarding behaviour. Animal Behaviour 26:707-711.

Auger, J., S. E. Meyer, and S. H. Jenkins. 2016. A mast-seeding desert shrub regulates population dynamics and behavior of its heteromyid dispersers. Ecology and Evolution 6:2275-2296.

Balda, R. P., and A. C. Kamil. 1992. Long-term spatial memory in Clark's nutcracker, Nucifraga columbiana. Animal Behaviour 44: 761-769.

Barkley, C. L., and L. F. Jacobs, L. F. 1998. Visual environment and delay affect cache retrieval accuracy in a food-storing rodent. Animal Learning \& Behavior 26:439-447.

Bednekoff, P. A., R. P. Balda, A. C. Kamil, and A. G. Hile. 1997. Long-term spatial memory in four seedcaching corvid species. Animal Behaviour 53:335-341.

Bogdziewicz, M., R. Zwolak, and E. E. Crone. 2016. How do vertebrates respond to mast seeding? Oikos 125:300-307.

Briggs, J. S., S. B. Vander Wall, and S. H. Jenkins. 2009. Forest rodents provide directed dispersal of Jeffrey pine seeds. Ecology 90:675-687.

Curran, L., and M. Leighton. 2000. Vertebrate responses to spatiotemporal variation in seed production of mast-fruiting Dipterocarpaceae. Ecological Monographs 70:101-128.

Dally, J. M., N. S. Clayton, and N. J. Emery. 2006a. The behaviour and evolution of cache protection and pilferage. Animal Behaviour 72:13-23.

Dally, J. M., N. J. Emery, and N. S. Clayton. 2006b. Food-caching western scrub-jays keep track of who was watching when. Science 312:1662-1665.

Dittel, J. W., R. Perea, and S. B. Vander Wall. 2017. Reciprocal pilfering in a seed-caching rodent community: implications for species coexistence. Behavioral Ecology and Sociobiology 71:147.

Dittel, J. W., and S. B. Vander Wall. 2018. Effects of rodent abundance and richness on cache pilfering. Integrative Zoology 13:331-338. 
Drobyshev, I., M. Niklasson, M. J. Mazerolle, and Y. Bergeron. 2014. Reconstruction of a 253-year long mast record of European beech reveals its association with large scale temperature variability and no long-term trend in mast frequencies. Agricultural and Forest Meteorology 192:9-17.

Forget, P. M., and S. B. Vander Wall. 2001. Scatter-hoarding rodents and marsupials: convergent evolution on diverging continents. Trends in Ecology \& Evolution 16:65-67.

Galvez, D., B. Kranstauber, R. W. Kays, and P. A. Jansen. 2009. Scatter hoarding by the Central American agouti: a test of optimal cache spacing theory. Animal Behaviour 78:1327-1333.

Gillespie, J. H. 1977. Natural selection for variances in offspring numbers: a new evolutionary principle. American Naturalist, 111:1010-1014.

Gittleman, J. L., and S. D. Thompson. 1988. Energy allocation in mammalian reproduction. American Zoologist 28:863-875.

Gu, H., Q. Zhao, and Z. Zhang. 2017. Does scatter-hoarding of seeds benefit cache owners or pilferers? Integrative Zoology 12:477-488.

Gómez, J. M., E. W. Schupp, and P. Jordano. 2019. Synzoochory: the ecological and evolutionary relevance of a dual interaction. Biological Reviews 94:874-902.

Grodziński, W., and K. Sawicka-Kapusta. 1970. Energy values of tree-seeds eaten by small mammals. Oikos 1:52-58.

Herrera, C. M., P. Jordano, J. Guitián, and A. Traveset. 1998. Annual variability in seed production by woody plants and the masting concept: reassessment of principles and relationship to pollination and seed dispersal. American Naturalist 152:576-594.

Hirsch, B.T., R. Kays, V. E. Pereira, and P. A. Jansen. 2012. Directed seed dispersal towards areas with low conspecific tree density by a scatter-hoarding rodent. Ecology Letters 15:1423-1429.

Hirsch, B.T., R. Kays, and P. A. Jansen. 2013. Evidence for cache surveillance by a scatter-hoarding rodent. Animal Behaviour 85:1511-1516. 
Jacobs, L. F., and E. R. Liman. 1991. Grey squirrels remember the locations of buried nuts. Animal Behaviour 41:103-110.

Jacobs, L. F. 1992. Memory for cache locations in Merriam's kangaroo rats. Animal Behaviour 43:585593.

Jansen, P. A., F. Bongers, and L. Hemerik. 2004. Seed mass and mast seeding enhance dispersal by a neotropical scatter-hoarding rodent. Ecological Monographs 74:569-589.

Jansen, P. A., B. T. Hirsch, W. J. Emsens, V. Zamora-Gutierrez, M. Wikelski, and R. Kays. 2012. Thieving rodents as substitute dispersers of megafaunal seeds. Proceedings of the National Academy of Sciences 109:12610-12615.

Jensen, T. S. 1985. Seed-seed predator interactions of European beech, Fagus silvatica and forest rodents, Clethrionomys glareolus and Apodemus flavicollis. Oikos 44:149-156.

Jones, K. E., J. Bielby, M. Cardillo, S. A. Fritz, J. O'Dell, C. D. L. Orme..., and C. Connolly. 2009. PanTHERIA: a species-level database of life history, ecology, and geography of extant and recently extinct mammals: Ecological Archives E090-184. Ecology 90:2648-2648.

Kantorowicz, W. 2000. Half a century of seed years in major tree species of Poland. Silvae Genetica $49: 245-248$.

Kelly, D. 1994. The evolutionary ecology of mast seeding. Trends in Ecology \& Evolution 9:465-470.

Kelly, D., and V. L. Sork. 2002. Mast seeding in perennial plants: why, how, where? Annual Review of Ecology and Systematics 33:427-447.

Koenig, W. D., and J. M. Knops. 2000. Patterns of annual seed production by northern hemisphere trees: a global perspective. American Naturalist 155:59-69.

Leaver, L. A., L. Hopewell, C. Caldwell, and L. Mallarky. 2007. Audience effects on food caching in grey squirrels (Sciurus carolinensis): evidence for pilferage avoidance strategies. Animal Cognition 10:23-27.

Lewontin, R. C., and D. Cohen. 1969. On population growth in a randomly varying environment. Proceedings of the National Academy of Sciences 62:1056-1060. 
Macdonald, D. W., and F. T. Tattersall. 2001. Britain's mammals - the challenge for conservation. Peoples Trust For Endangered Species, London.

Meyer, S. E., and B. K. Pendleton. 2015. Evolutionary drivers of mast-seeding in a long-lived desert shrub. American Journal of Botany 102:1666-1675.

Mendoza, I., R. S. Condit, S. J. Wright, A. Caubère, P. Châtelet, I. Hardy, and P. M. Forget. 2018. Interannual variability of fruit timing and quantity at Nouragues (French Guiana): insights from hierarchical Bayesian analyses. Biotropica 50:431-441.

Millar, J. S. 1978. Energetics of reproduction in Peromyscus leucopus: the cost of lactation. Ecology 59:1055-1061.

Millar, J. S. 1979. Energetics of lactation in Peromyscus maniculatus. Canadian Journal of Zoology 57:1015-1019.

Muñoz, A., and R. Bonal. 2011. Linking seed dispersal to cache protection strategies. Journal of Ecology 99:1016-1025.

Norden, N., J. Chave, P. Belbenoit, A. Caubère, P. Châtelet, P. M. Forget, and C. Thébaud. 2007. Mast fruiting is a frequent strategy in woody species of Eastern South America. PLoS One 2:e1079.

Ostfeld, R. S., and F. Keesing. 2000. Pulsed resources and community dynamics of consumers in terrestrial ecosystems. Trends in Ecology \& Evolution 15:232-237.

Övergaard, R., P. Gemmel, and M. Karlsson. 2007. Effects of weather conditions on mast year frequency in beech (Fagus sylvatica L.) in Sweden. Forestry, 80:555-565.

Paar, U., A. Guckland, I. Dammann, M. Albrecht, J. Eichhorn. 2011. Häufigkeit und Intensität der Fruktifikation der Buche [Frequency and intensity of fruiting in beech]. AFZ-DerWald 6:26-29.

Pearse, I. S., W. D. Koenig, and D. Kelly. 2016. Mechanisms of mast seeding: resources, weather, cues, and selection. New Phytologist 212:546-562.

Pearse, I. S., J. M. LaMontagne, and W. D. Koenig. 2017. Inter-annual variation in seed production has increased over time (1900-2014). Proceedings of the Royal Society of London B: Biological Sciences 284:20171666. 
Pesendorfer, M. B., T. S. Sillett, W. D. Koenig, and S. A. Morrison. 2016. Scatter-hoarding corvids as seed dispersers for oaks and pines: a review of a widely distributed mutualism and its utility to habitat restoration. Condor 118:215-237.

Pravosudov, V. V., T. C. Roth II, and L. D. LaDage. 2010. Chickadees are selfish group members when it comes to food caching. Animal Behaviour 80:175-180.

Pucek, Z. 1984. Klucz do oznaczania ssaków Polski. Państwowe Wydawnictwo Naukowe, Warszawa.

Pucek, Z., W. Jędrzejewski, B. Jędrzejewska, and M. Pucek. 1993. Rodent population dynamics in a primeval deciduous forest (Białowieża National Park) in relation to weather, seed crop, and predation. Acta Theriologica 38:199-232.

Schauber, E. M., D. Kelly, P. Turchin, C. Simon, W. G. Lee, R. B. Allen, I. J. Payton, P. R. Wilson, P. E. Cowan, and R. E. Brockie. 2002. Masting by eighteen New Zealand plant species: the role of temperature as a synchronizing cue. Ecology 83:1214-1225.

Schmidt, K. A., and R. S. Ostfeld. 2008. Eavesdropping squirrels reduce their future value of food under the perceived presence of cache robbers. American Naturalist 171:386-393.

Schmidt, W. 2006. Temporal variation in beech masting (Fagus sy/vatica L.) in a limestone beech forest (1981-2004). Allgemeine Forst und Jagdzeitung 177:9-19.

Schreiber, S. J. 2015. Unifying within-and between-generation bet-hedging theories: an ode to JH Gillespie. American Naturalist 186:792-796.

Shaw, R. C., and N. S. Clayton. 2013. Careful cachers and prying pilferers: Eurasian jays (Garrulus glandarius) limit auditory information available to competitors. Proceedings of the Royal Society of London B: Biological Sciences 280:20122238.

Shibata, M., H. Tanaka, S. lida, S. Abe, T. Masaki, K. Niiyama, and T. Nakashizuka. 2002. Synchronized annual seed production by 16 principal tree species in a temperate deciduous forest, Japan. Ecology 83:1727-1742.

Sikes, R. S. 1995. Costs of lactation and optimal litter size in northern grasshopper mice (Onychomys leucogaster). Journal of Mammalogy 76:348-357. 
Smulders, T. V. 1998. A game theoretical model of the evolution of food hoarding: applications to the Paridae. American Naturalist 151:356-366.

Steele, M. A., S. L. Halkin, P. D. Smallwood, T. J. McKenna, K. Mitsopoulos, and M. Beam. 2008. Cache protection strategies of a scatter-hoarding rodent: do tree squirrels engage in behavioural deception? Animal Behaviour 75:705-714.

Steele, M. A., M. Bugdal, A. Yuan, A. Bartlow, J. Buzalewski, N. Lichti, and R. Swihart. 2011. Cache placement, pilfering, and a recovery advantage in a seed-dispersing rodent: could predation of scatter hoarders contribute to seedling establishment? Acta Oecologica 37:554-560.

Steele, M. A., T. A. Contreras, L. Z. Hadj-Chikh, S. J. Agosta, P. D. Smallwood, and C. N. Tomlinson. 2013. Do scatter hoarders trade off increased predation risks for lower rates of cache pilferage? Behavioral Ecology 25:206-215.

Steele, M. A., G. Rompre, J. A. Stratford, H. Zhang, M. Suchocki, and S. Marino. 2015. Scatterhoarding rodents favor higher predation risks for cache sites: the potential for predators to influence the seed dispersal process. Integrative Zoology 10:257-266.

Thayer, T. C., and S. B. Vander Wall. 2005. Interactions between Steller's jays and yellow pine chipmunks over scatter-hoarded sugar pine seeds. Journal of Animal Ecology 74:365-374.

Vander Wall, S. B. 2001. The evolutionary ecology of nut dispersal. Botanical Review 67:74-117.

Vander Wall, S. B. 2002. Masting in animal-dispersed pines facilitates seed dispersal. Ecology

$$
\text { 83:3508-3516. }
$$

Vander Wall, S. B. 2010. How plants manipulate the scatter-hoarding behaviour of seed-dispersing animals. Philosophical Transactions of the Royal Society B 365:989-997.

Vander Wall, S. B., and S. H. Jenkins. 2003. Reciprocal pilferage and the evolution of food-hoarding behavior. Behavioral Ecology 14:656-667.

Vander Wall, S. B., J. S. Briggs, S. H. Jenkins, K. M. Kuhn, T. C. Thayer, and M. J. Beck. 2006. Do foodhoarding animals have a cache recovery advantage? Determining recovery of stored food. Animal Behaviour 72:189-197. 
597 Vander Wall, S. B., C. J. Downs, M. S. Enders, and B. A. Waitman. 2008. Do yellow-pine chipmunks

598 prefer to recover their own caches? Western North American Naturalist 68:319-324.

599 Vander Wall, S. B., S. C. Barga, and A. E. Seaman. 2017. The geographic distribution of seed-dispersal 600 mutualisms in North America. Evolutionary Ecology 31:725-740.

601 Yang, L. H., K. F. Edwards, J. E. Byrnes, J. L. Bastow, A. N. Wright, and K. O. Spence. 2010. A meta602 analysis of resource pulse-consumer interactions. Ecological Monographs 80:125-151.

603 Zhu, W., Y. Mu, J. Liu, and Z. Wang. 2015. Energy requirements during lactation in female Apodemus 604 chevrieri (Mammalia: Rodentia: Muridae) in the Hengduan Mountain region, Italian Journal of $605 \quad$ Zoology 82:2.

606 Zwolak, R., M. Bogdziewicz, A. Wróbel, and E. E. Crone. 2016. Advantages of masting in European 607 beech: timing of granivore satiation and benefits of seed caching support the predator dispersal 608 hypothesis. Oecologia 180:749-758. 


\section{Appendix. Derivation of the fitness gradient}

To derive the fitness gradient, assume that the population dynamics of individuals with the resident threshold strategy $T$ converges to a periodic solution of period $k P: n_{i}(1), n_{i}(2), \ldots, n_{i}(p)$ for $i=$ $1,2,3$. The more general case of aperiodic dynamics is discussed at the end of this appendix. For each $1 \leq t \leq k P$, let $G(t)$ be the amount seeds gathered in Fall by each resident individual in year $t$, and $Q_{1}(t), Q_{2}(t), Q_{3}(t)$ be the number of offspring produced by an individual playing the mutant strategy $T_{m}$ during the Fall, Winter/Spring, and Summer, respectively, in year $t$. Then, the long-term per-capita growth rate of the mutant is

$$
r\left(T, T_{m}\right)=\frac{1}{k P} \sum_{t=1}^{k P} \sum_{i=1}^{3} \log \left(Q_{i}(t)+s_{i}\right)
$$

Taking the derivative with respect to $T_{m}$ and evaluating at $T_{m}=T$ gives us the fitness gradient:

$$
\frac{\partial r}{\partial T_{m}}(T, T)=\frac{1}{k P} \sum_{t=1}^{k P} \sum_{i=1}^{3} \frac{\left.\frac{\partial Q_{i}(t)}{\partial T_{m}}\right|_{T_{m}=T}}{\left.Q_{i}(t)\right|_{T_{m}=T}+s_{i}}
$$

As $\left.Q_{i}(t)\right|_{T_{m}=T}$ corresponds to the fecundity of resident individuals,

$$
\left.Q_{i}(t)\right|_{T_{m}=T}=R_{i}(t)
$$

It remains to compute the partial derivatives of the $Q_{i}(t)$ terms. For $i=1$, we have (from the main text)

$$
Q_{1}(t)=\frac{b \min \left\{G(t), T_{m}\right\}}{h+\min \left\{G(t), T_{m}\right\}}
$$

is piecewise defined depending on whether $G(t)<T$ or $>T$. Specifically, we get

$$
\left.\frac{\partial Q_{1}(t)}{\partial T_{m}}\right|_{T_{m}=T}= \begin{cases}0 & \text { if } T>G(t) \\ \frac{b h}{(h+T)^{2}} & \text { if } T<G(t) .\end{cases}
$$


For $i=2$, we have (from the main text)

$$
Q_{2}(t)=\frac{b C_{\mathrm{m}, \text { survivor }}(t)}{h+C_{\mathrm{m}, \text { survivor }}(t)} \frac{s_{1}}{Q_{1}(t)+s_{1}}+\frac{b C_{\text {new }}(t)}{h+C_{\text {new }}(t)} \frac{Q_{1}(t)}{Q_{1}(t)+s_{1}}
$$

where $Q_{2}(t)$ differs from $R_{2}(t)$ only in its first term due to surviving individuals with the mutant caching strategy:

$C_{\mathrm{m}, \text { survivor }}(t)=\max \left\{G(t)-T_{m}, 0\right\} M(t)+\max \{G(t)-T, 0\}\left(O(t)\left(s_{1} n_{1}(t)-1\right)+D(t)\left(1-s_{1}\right) n_{1}(t)\right)$.

Hence, by chain rule,

$$
\left.\frac{\partial Q_{2}(t)}{\partial T_{m}}\right|_{T_{m}=T}=\left.\frac{b h}{\left(h+C_{\mathrm{m}, \text { survivor }}(t)\right)^{2}} \frac{s_{1}}{Q_{1}(t)+s_{1}} \frac{\partial C_{\mathrm{m}, \text { survivor }}(t)}{\partial T_{m}}\right|_{T_{m}=T}
$$

At $T_{m}=T$, we have $C_{\mathrm{m}, \text { survivor }}(t)=C_{\text {survivor }}(t)$ and $Q_{1}(t)=R_{1}(t)$. Furthermore,

$$
\left.\frac{\partial C_{\mathrm{m}, \text { survivor }}(t)}{\partial T_{m}}\right|_{T_{m}=T}= \begin{cases}0 & \text { if } T>G(t) \\ -M(t) & \text { if } T<G(t)\end{cases}
$$

Thus,

$$
\left.\frac{\partial Q_{2}(t)}{\partial T_{m}}\right|_{T_{m}=T}= \begin{cases}0 & \text { if } T>G(t) \\ -\frac{b h}{\left(h+C_{\text {survivor }}(t)\right)^{2}} \frac{s_{1}}{R_{1}(t)+s_{1}} M(t) & \text { if } T<G(t) .\end{cases}
$$

Finally, as discussed in the main text, $Q_{3}(t)=R_{3}(t)$ and, consequently, doesn't depend on $T_{m}$. Hence,

$$
\left.\frac{\partial Q_{3}(t)}{\partial T_{m}}\right|_{T_{m}=T}=0
$$

Substituting equations (A2)-(A5) into (A1) provides an explicit expression for the fitness gradient.

These calculations also apply to the more general case when the resident dynamics are asymptotically stationary but not necessarily periodic. By asymptotic stationarity, we mean that there 
exists a probability measure $\mu$ on the $\left(S, n_{1}\right)$ state space such that

$$
\lim _{T \rightarrow \infty} \frac{1}{T} \sum_{t=1}^{T} f\left(S(t), n_{1}(t)\right)=\int f\left(S, n_{1}\right) \mu\left(d S, d n_{1}\right)
$$

for all continuous functions $f\left(S, n_{1}\right)$ i.e. the Birkhoff averages converge. In this case, the per-capita growth rate of the mutant is well-defined and equals

$$
r\left(T, T_{m}\right)=\lim _{T \rightarrow \infty} \frac{1}{T} \sum_{t=1}^{T} \sum_{i=1}^{3} \log \left(Q_{i}(t)+s_{i}\right) .
$$

The selection gradient equals

$$
\frac{\partial r}{\partial T_{m}}(T, T)=\lim _{T \rightarrow \infty} \frac{1}{T} \sum_{t=1}^{T} \sum_{i=1}^{3} \frac{\left.\frac{\partial Q_{i}(t)}{\partial T_{m}}\right|_{T_{m}=T}}{\left.Q_{i}(t)\right|_{T_{m}=T}+s_{i}}
$$

and can be explicitly evaluated by using (A2)-(A5). 